\title{
Perineal use of talc and risk of ovarian cancer
}

\author{
H Langseth, ${ }^{1}$ S E Hankinson, ${ }^{2}$ J Siemiatycki, ${ }^{3}$ E Weiderpass ${ }^{1,4,5}$
}

${ }^{1}$ The Cancer Registry of

Norway, Institute of populationbased Cancer Research, Oslo,

Norway; ${ }^{2}$ Channing Laboratory, Department of Medicine,

Brigham and Women's Hospital and Harvard Medical School,

Boston, MA, USA; ${ }^{3}$ Department of Social and Preventive

Medicine, University of

Montreal, Montreal, Canada;

${ }^{4}$ Department of Medical

Epidemiology and Biostatistics,

Karolinska Institutet, Stockholm,

Sweden; ${ }^{5}$ Samfundet

Folkhälsan, Helsinki, Finland

Correspondence to:

E Weiderpass, The Cancer

Registry of Norway, 0310 Oslo,

Norway; eliwei@ki.se

Accepted 15 October 2007

\section{ABSTRACT}

Ovarian cancer is one of the most common gynaecological neoplasms, especially in industrialised countries. The aetiology of the disease is not well understood, except that inherited mutations in the breast cancer genes BRCA1 and BRCA-2 account for up to $10 \%$ of all cases, ${ }^{1}$ and child-bearing, oral contraceptive use and breast-feeding reduce the risk. ${ }^{2}$ Some environmental exposures, notably talc and asbestos, have been suspected as ovarian carcinogens.

Talc refers to both mineral talc and industrial products that contain mineral talc. Mineral talc occurs naturally in many regions of the world and is valued for its softness, platyness, and ability to absorb organic matter. Mineral talc occurs naturally in a platy (flat) form, but may also occur as asbestiform fibres, which describes its physical form and does not imply the presence of asbestos. The purer forms (approximately 90\% mineral talc) are used for cosmetic and hygiene products including baby powders and feminine hygiene products. Perineal use of cosmetic talc is a common practice in the United Kingdom, North America, Australia and some other countries. To our knowledge accurate estimates of prevalence of use of cosmetic talc are not available. However, the use for female hygiene of body powders, baby powders, talcum powders and deodorising powder, most of which contain cosmetic talc in varying amounts, has been reported to be as high as $50 \%$ in some countries. ${ }^{3}$

From pathological studies it is known that particles and fibres that enter the body can migrate to distant organs. For instance, asbestos fibres have been found in ovaries from women exposed to asbestos. ${ }^{45}$ Analogously, following perineal application, talc particles can migrate from the vagina to the peritoneal cavity and ovaries. ${ }^{6}$ A majority of women experience retrograde menstruation ${ }^{7}$; this suggests a mechanism by which talc particles can travel through the female reproductive tract to the ovaries. Furthermore, epidemiological studies have shown decreased risks of ovarian cancer after tubal ligation and/or hysterectomy, suggesting that removing a pathway by which carcinogenic substances can reach the ovaries reduces the risk. ${ }^{89}$

The association between talc use in the perineal region and ovarian cancer was investigated in one cohort study, ${ }^{10}$ and 20 case-control studies. ${ }^{11-30}$ In the cohort study, arguably the strongest study because of its partly prospective ascertainment of exposure, there was no association between cosmetic talc use and risk of all subtypes of ovarian cancer combined. The various case-control studies provided indications of either a significant excess risk (10 studies) or non-significant excess risk or null (10 studies), with odds ratios (ORs) ranging from 1.0 to 3.9. None of the studies reported relative risks below 1.0. The population-based casecontrol studies, ${ }^{11} 15-17$ 20-26 28-30 included studies with 112-824 ovarian cancer cases, and had odds ratios ranging from 1.1 to 3.9 (fig 1). The hospital-based case control studies ${ }^{12-14} 181927$ included studies with 77-462 cases, and reported odds ratios between 1.0 and 2.5. Pooled odds ratios were calculated by fixed effects model. As shown in figure 1 pooled ORs were 1.40, 1.12 and 1.35 for population-based, hospital-based and all case control studies combined, respectively. Some studies ${ }^{13} 1422232628$ tried to assess exposure-response associations, in terms of frequency of use or length of use in years but found no clear trend.

Methodological factors such as recall bias should always be considered in case-control studies. It could have been a problem had there been widespread publicity about the possible association between use of body powder and cancer. The International Agency for Research on Cancer (IARC) working group considers that there has not been widespread public concern about this issue and therefore considers it unlikely that such a bias could explain the consistent findings. Another source of recall bias could result from the fact that women with the cancer tend to remember or overreport their use of body powder. The influence of this type of recall bias cannot be ruled out.

Eight of the population-based case-control stu$\operatorname{dies}^{11} 1622-24262829$ were identified, by the IARC working group as being most informative in terms of size of the studies, whether the studies were population-based, participation rates and adjustments of confounding variables. The selected studies included at least 188 cases and had participation rates ranging from $60 \%$ to $75 \%$. Among these eight studies, the prevalence of perineal use of talc-based body powder among controls ranged from 16\% to $52 \%$. The relative risks of ovarian cancer among body powder users were homogeneous across this set of eight studies, each of which indicated a 30 $60 \%$ increase in risk. Among the other 12 casecontrol studies, most also reported relative risks of this magnitude or higher.

Information on talc use in infancy is generally insufficient in the case-control studies. However, in one study the exposure to baby powder was reported by $42.2 \%$ of the cases and $40.5 \%$ of the controls. ${ }^{15}$ In several of the other studies patients were asked about age at first use of perineal talc, as an indicator for use in infancy or other periods of life.

Only four case-control studies ${ }^{16232930}$ and one cohort study ${ }^{10}$ provided results by histological type. In four of these studies, in particular the cohort study, there were hints of higher risks of serous tumours related to talc exposure. 


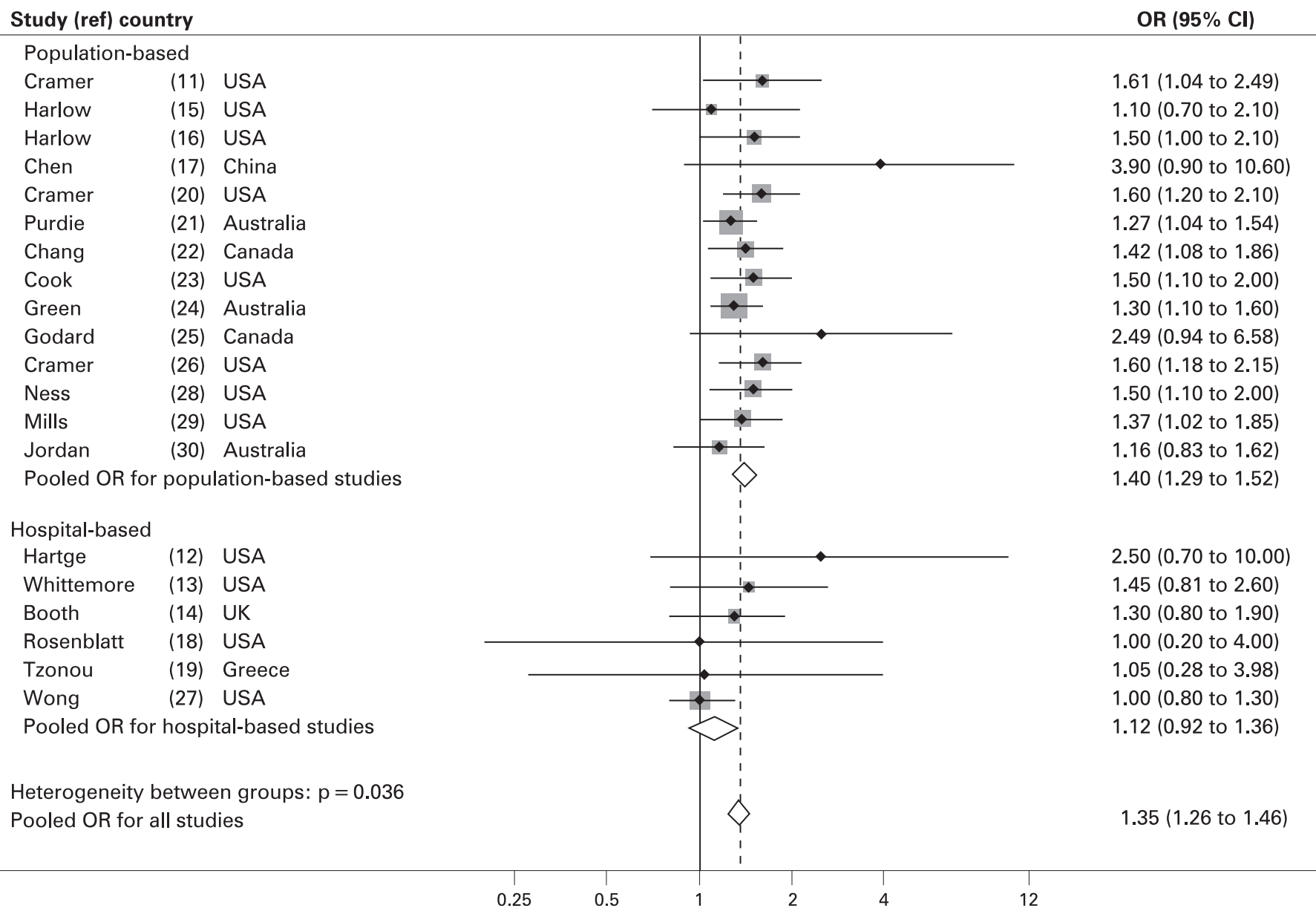

Figure 1 Results from case-control studies contributing data on perineal talc use and ovarian cancer. Results are presented as odds ratios (ORs) and their corresponding confidence intervals (95\% Cls) and represented by squares and lines, respectively. Results are separated in 14 population-based and six hospital-based case-control studies. Pooled ORs for all population-based studies combined and all hospital-based studies combined are given. OR pooling by fixed effect models (Mantel-Haenszel method).

Before 1976, talc was to some extent contaminated with asbestos, so that the early studies relating talc to ovarian cancer may have been confounded by the asbestos. ${ }^{31}$ However, the association between talc exposure and ovarian cancer is as strong in recent studies, ${ }^{28}{ }^{29}$ as in earlier ones, diminishing the likelihood that all these results are influenced by contamination of talc by asbestos.

To summarise the evidence in favour of an association, a very large number of studies have found that women who used talc experienced excess risks of ovarian cancer; some results were statistically significant and some were not. There was some indication in the cohort study of an increase in serous tumours. The evidence of talc migrating to the ovaries lends credibility to such a possible association. The main epidemiological evidence against the association is the absence of clear exposure-response associations in most studies, as well as the absence of an overall excess risk in the cohort study.

On balance, the epidemiological evidence suggests that use of cosmetic talc in the perineal area may be associated with ovarian cancer risk. The mechanism of carcinogenicity may be related to inflammation. ${ }^{32}$

The carcinogenicity of non-asbestiform talc was assessed by a monograph working group at IARC in $2006 .{ }^{33}$ After considering biases and possible confounding factors, the IARC working group concluded that the epidemiological studies provided limited evidence for the carcinogenicity of perineal use of talcbased body powder, and classified this use as possibly carcinogenic to human beings (that is, group $2 \mathrm{~B}$ ). ${ }^{34}$

\section{PROPOSAL: TO RESEARCH COMMUNITY}

The current body of experimental and epidemiological evidence is insufficient to establish a causal association between perineal use of talc and ovarian cancer risk. Experimental research is needed to better characterise deposition, retention and clearance of talc to evaluate the ovarian carcinogenicity of talc.

The majority of the epidemiological studies carried out so far have been among American women. It would be instructive to seek evidence in other countries where perineal use of talc has been common.

While there has been some efforts to measure the degree of use, these have mainly been measured simply as the reported years of use. It is possible that the ostensible lack of exposure response trends is the result of crudeness of the exposure metric used. Therefore, it is important that future studies, irrespective of study design, devote some effort to better assessment of exposure. The use of body powders should be assessed both in terms of calendar time and age of the subject. Subjects should be asked about lifetime use, including age at initial use (infancy, childhood, teenager years, adulthood), age at which they stopped using such powders, gaps in the lifetime period of use 


\section{What this study adds}

- Epidemiological evidence suggests that use of cosmetic talc in the perineal area may be associated with ovarian cancer risk. The IARC has classified this use of talc as possibly carcinogenic to human beings (group 2B).

- The mechanism of carcinogenicity may be related to inflammation. This paper focus on the high degree of consistency in the studies accomplished so far, and what should be the focus in future studies.

and frequency and nature of use (daily, during certain seasons of the year, only while menstruating). Another important question is whether the use of body powder was before or after tubal ligation or hysterectomy.

Individuals' answers to questions about use of brand names over time may be unreliable, and therefore, in future studies, investigators should try to ascertain, either from government or industry sources, the composition of the powders used in different time periods by different brand names and, in particular, to ascertain whether the exposure may have included some contamination by asbestos and also whether the exposure was to talc or a non-talc product. Statistical analyses should attempt to assess risk separately for the categories of powders: talc containing asbestos, talc not containing asbestos, non-talc product. Further, exposure metrics should take into account the age, duration and intensity of exposure. As well as analyses for all ovarian tumours combined, there should, if possible, be analyses by histological subtype and by invasiveness of the tumour.

While it would not be reasonable to envisage establishing a costly long-term prospective cohort study just to study this association, any long-term cohort study that is being set up to study cancer among women should collect information about talc use if the study is being conducted in a country where such use has been widespread.

In summary, future studies should focus on seeking evidence in talc-exposed female populations worldwide, collecting reliable information on age at initial use of body powder, exposure assessments and dose response associations.

Acknowledgements: The work reported in this paper was initiated while SH, JS and EW were part of an IARC Monographs Working Group of the International Agency for Research on Cancer, Lyon, France.

Funding: This study was financed by the Cancer Registry of Norway.

Competing interests: None.

\section{REFERENCES}

1. Lux MP, Fasching PA, Beckmann MW. Hereditary breast and ovarian cancer: review and future perspectives. J Mol Med 2006;84:16-28.

2. Persson I. Estrogens in the causation of breast, endometrial and ovarian cancersevidence and hypotheses from epidemiological findings. J Steroid Biochem Mol Biol 2000;74:357-64.
3. International Agency for Research on Cancer. IARC monographs on the evaluation of carcinogenic risks to human, Vol 93, Carbon black, titanium dioxide, and non-asbestiform talc. Geneva: WHO (in press).

4. Heller DS, Gordon RE, Westhoff $C$, et al. Asbestos exposure and ovarian fiber burden. Am J Ind Med 1996;29:435-9.

5. Langseth $\mathbf{H}$, Johansen BV, Nesland JM, et al. Asbestos fibers in ovarian tissue from Norwegian pulp and paper workers. Int J Gynecol Cancer 2007;17:44-9.

6. Venter PF. Ovarian epithelial cancer and chemical carcinogenesis. Gynecol Oncol 1981;12:281-5.

7. Olive DL, Schwartz LB. Endometriosis. N Eng/ J Med 1993;328:1759-69.

8. Rosenblatt KA, Thomas DB. Reduced risk of ovarian cancer in women with a tubal ligation or hysterectomy. the World Health Organization Collaborative Study of Neoplasia and Steroid Contraceptives. Cancer Epidemiol Biomarkers Prev 1996;5:933-5.

9. Tortolero-Luna G, Mitchell MF. The epidemiology of ovarian cancer. J Cell Biochem Supp/ 1995;23:200-7.

10. Gertig DM, Hunter DJ, Cramer DW, et al. Prospective study of talc use and ovarian cancer. J Natl Cancer Inst 2000;92:249-52.

11. Cramer DW, Welch WR, Scully RE, et al. Ovarian cancer and talc: a case-control study. Cancer 1982;50:372-6.

12. Hartge $\mathbf{P}$, Hoover $\mathrm{R}$, Lesher $\mathrm{LP}$, et al. Talc and ovarian cancer. JAMA 1983;250:1844

13. Whittemore AS, Wu ML, Paffenbarger RS Jr, et al. Personal and environmental characteristics related to epithelial ovarian cancer. II. Exposures to talcum powder, tobacco, alcohol, and coffee. Am J Epidemiol 1988;128:1228-40.

14. Booth M, Beral V, Smith P. Risk factors for ovarian cancer: a case-control study. Br J Cancer 1989;60:592-8.

15. Harlow BL, Weiss NS. A case-control study of borderline ovarian tumors: the influence of perineal exposure to talc. Am J Epidemiol 1989;130:390-4.

16. Harlow BL, Cramer DW, Bell DA, et al. Perineal exposure to talc and ovarian cancer risk. Obstet Gynecol 1992;80:19-26.

17. Chen Y, Wu PC, Lang JH, et al. Risk factors for epithelial ovarian cancer in Beijing, China. Int J Epidemiol 1992;21:23-9.

18. Rosenblatt KA, Szklo M, Rosenshein NB. Mineral fiber exposure and the development of ovarian cancer. Gynecol Oncol 1992;45:20-5.

19. Tzonou A, Polychronopoulou A, Hsieh CC, et al. Hair dyes, analgesics, tranquilizers and perineal talc application as risk factors for ovarian cancer. Int $J$ Cancer 1993:55:408-10.

20. Cramer DW, Xu H. Epidemiologic evidence for uterine growth factors in the pathogenesis of ovarian cancer. Ann Epidemiol 1995;5:310-4.

21. Purdie D, Green A, Bain C, et al. Reproductive and other factors and risk of epithelial ovarian cancer: an Australian case-control study. Survey of Women's Health Study Group. Int J Cancer 1995;62:678-84.

22. Chang S, Risch HA. Perineal talc exposure and risk of ovarian carcinoma. Cancer 1997; 79:2396-401.

23. Cook LS, Kamb ML, Weiss NS. Perineal powder exposure and the risk of ovarian cancer. Am J Epidemiol 1997;145:459-65.

24. Green A, Purdie D, Bain C, et al. Tubal sterilisation, hysterectomy and decreased risk of ovarian cancer. Survey of Women's Health Study Group. Int J Cancer 1997; 71:948-51.

25. Godard B, Foulkes WD, Provencher D, et al. Risk factors for familial and sporadic ovarian cancer among French Canadians: a case-control study. Am J Obstet Gynecol 1998;179:403-10.

26. Cramer DW. Perineal talc exposure and subsequent epithelial ovarian cancer: a case-control study. Obstet Gynecol 1999;94:160-1.

27. Wong C, Hempling RE, Piver MS, et al. Perineal talc exposure and subsequent epithelial ovarian cancer: a case-control study. Obstet Gynecol 1999;93:372-6.

28. Ness RB, Grisso JA, Cottreau C, et al. Factors related to inflammation of the ovarian epithelium and risk of ovarian cancer. Epidemiology 2000;11:111-7.

29. Mills PK, Riordan DG, Cress RD, et al. Perineal talc exposure and epithelial ovarian cancer risk in the Central Valley of California. Int J Cancer 2004;112:458-64.

30. Jordan SJ, Green AC, Whiteman DC, et al. Risk factors for benign serous and mucinous epithelial ovarian tumors. Obstet Gynecol 2007;109:647-54.

31. Harlow BL, Hartge PA. A review of perineal talc exposure and risk of ovarian cancer Regul Toxicol Pharmacol 1995;21:254-60.

32. Ness RB, Cottreau C. Possible role of ovarian epithelial inflammation in ovarian cancer. J Natl Cancer Inst 1999;91:1459-67.

33. International Agency for Research on Cancer. Preamble to the IARC monographs on the evaluation of carcinogenic risks to humans. 93. Lyon: IARC

34. Baan R, Straif K, Grosse Y, et al. Carcinogenicity of carbon black, titanium dioxide, and talc. Lancet Oncol 2006;7:295-6. 